\title{
Regulating emotions in young adulthood
}

Young adults with complex mental health needs often find it difficult Hall and her collaborators from Deakin University, Australia, have developed and evaluated an intervention that has applicability across diagnostic categories of mental health and substance use needs, and can be delivered alongside existing treatments. This intervention teaches young people how to manage their emotions and urges them to make good decisions in spite of emotional distress. The programm in achieve healthy developmental outcomes.

oung adulthood is a critical life
stage marked by social and
emotional challenges and by emotional challenges and by person navigates the demands and transitions of this complex developm phase. For adolescents exposed to childhood trauma and developmental stress, this vulnerability is particularly evident, as these early life experiences can reduce opportunities to develop skill and techniques for managing emotions. Adverse life experiences such as abuse, neglect, family conflict, and homeless rically mpact the development

NXIETr mental health disorders emerge adds to the significant vulnerability of this
vald to the signifan

vulnerability of this
cohort of young

cohort of young
people, many of whom access
primary mental health

舟

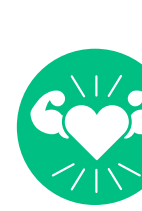

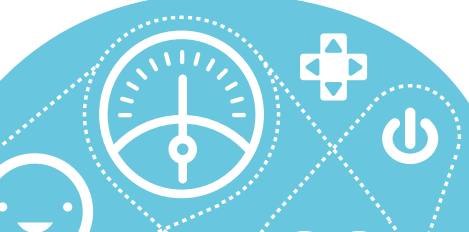

A transdiagnostic model considers the common psychological processes that underpin mental health disorders across diagnostic categories. These models offer a practical approach for managing the complexity with which young people present for treatment by simultaneously targeting psychologica processes that maintain distress actoss support gathered over decades of

treatment studies.

services for assistance with mental hea and substance use difficulties.

Dr Kate Hall and Dr Elise Sloan at Deakin University, Australia, say that treatment at youth services commonly have multiple mental health needs across diagnostic categories, there currently are few evidence-informed approaches that target the needs of this vulnerable cohort. Reasons for presenting to services may include problematic substance use or deliberate self-harm as well as vario other mental health disorders, such as and traits of borderline personal ity disorder Existing psychological treatment approaches rarely target more than one disorder and are not commonly developed with young people, for young people. These approaches often apply strategies to target symptoms of a spectic mental health disorder, without focusing on the interactive complexity of proces that may underlie the presentation of mental health distress. Dr Hall and Dr Sloan argue that new transdiagnostic approaches are required to address treatment ty and transoliagnostic are navigating the young people who challenges necessary for a healthy
SADNESS transition from adoless impact that engagement in maladaptive emotion regulation strategies (i.e.

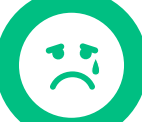

\section{EMOTON REGULATION AND}

Dr Hall and Dr Sloan have collaborated

on a six-year body of work, in

with youth services and their

research team, to examine emotion

healh and substance use conditions

in

which in is a mulidimensional concept

micro-skills involved in modusting and

managing the experience and expression of emotions. It includes an awareness,

emotions, the ability to apply appropria

strategies or skills to manage emotions,

and control the impulse to respond

to emotions in unhealthy ways when

experiencing negative emotions. The

research team explains that difficulties

werlying process evident in young

people who have histories of traung

health difficulties.

Dr Hall and Dr Sloan examined profiles of emotion regulation in treatmentproaches have significagn empicic

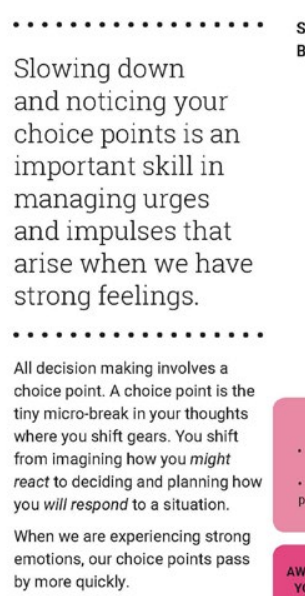

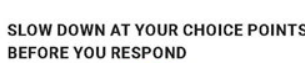

with emotion regulation are a common

\section{Diffeur srivuan}

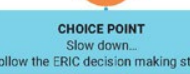

(iil) (1)

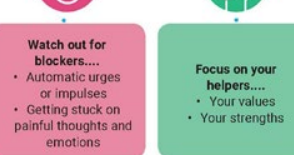

站聂自
The ERIC programme helps young people to

rumination, avoidance, and suppression) had on the severity of their mental hea systematic review of studies that measure changes in both emotion regulation and symptoms of psychopathology and found that the ability to effectively regulate emotions is something that can be taught through cognitive behavioural-based interventions. Consequently, the resear team have determined that emotion regulation is an important treatment target in transdiagnostic models that aim to address the complex patterns of mental health, substance use, and psychosocial difficulties that are often present in this group of young people. Taken together,
this research informed the Control (ERIC) is a transdiagnostic reatment model that builds on various theoretical

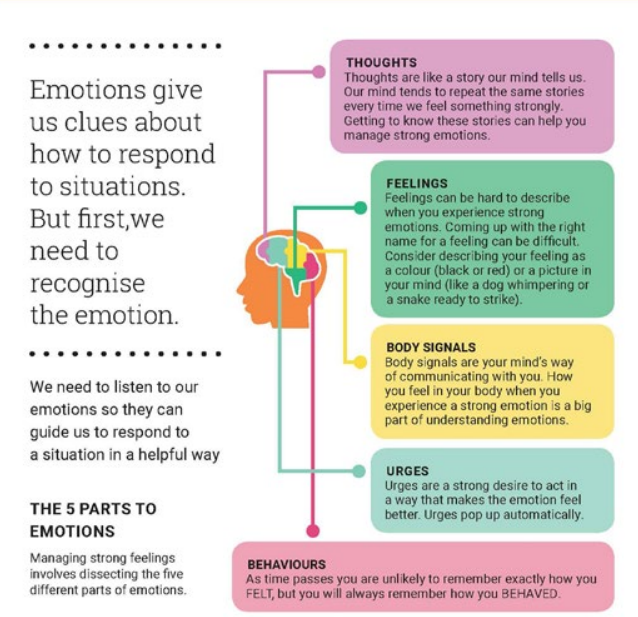

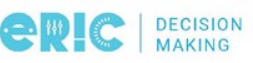

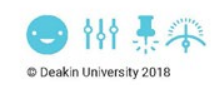

ERICI HO
Self kindness

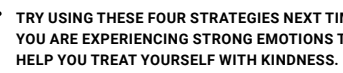

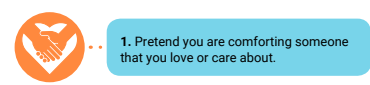

daily. Being
kind rather th

kind rather than
critical towards
ourselves when



we are suffering helps us mana

(3).

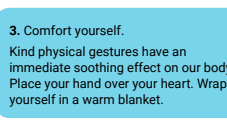

comforting ourseles with

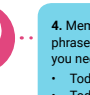

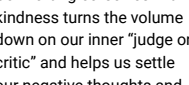

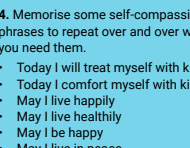

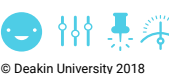
in eight domains of emotion regulation
and impulse control. Each of these and vulnerable young people who were accessing services for assistance with ERIC delivers emotion regulation microskills in a modular format, which means that it can be integrated and complement already existing interventions delivered in the youth sector, including case management, youth outreach support, life skills training
amongst others.

ERIC integrates and draws on existing theories of emotion regulation to deliver development of an models that aim to address the

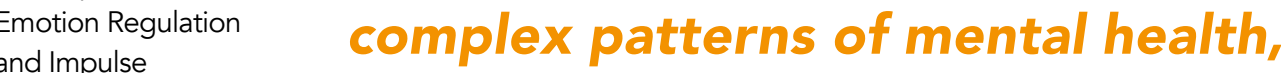
substance use and psychosocial difficulties in young people.

The theoretical models that inform Roemer's 2004 Multidude Gratz and of Emotion Dysregulation and Berking and Whitley's 2014 Adaptive Coping with Emotions Model. These models focus on enolional awareness and understanding enotional experiences and triggers, as emotions. ERIC also incorporated Gross's 998 Process Model Emotion Regulation which focuses on applying strategies for both before the onset of the response and in response to an event.

The research team models of emotion regulation and applies evidence-based strategies to promote healthy social and emotional development outcomes in young adults by helping them to build emotion regulation and impulse control skills.

domains has outcomes that represe healthy development. The skills and processes in each of these domains are delivered in a modular format and comprise practical exercises that includ psychoeducation delivered through analogy or mind mapping, behavio

The intervention was developed as part of schedules. The micro-skills cultivated three-year action research programme across all ight domains ultimately teach and incorporated evidence from studies together with the theoretical constructs underpinning the model conpirical cork on specifing the model included strategies such as rution regulation avoidance, reapramisation, suppression acceptance, problem-solving , and selfcompassion. The skills taught in ERIC include distress tols taught in ERIC 
EMOTIONAL REGULATION

IMPULSE CONTROL

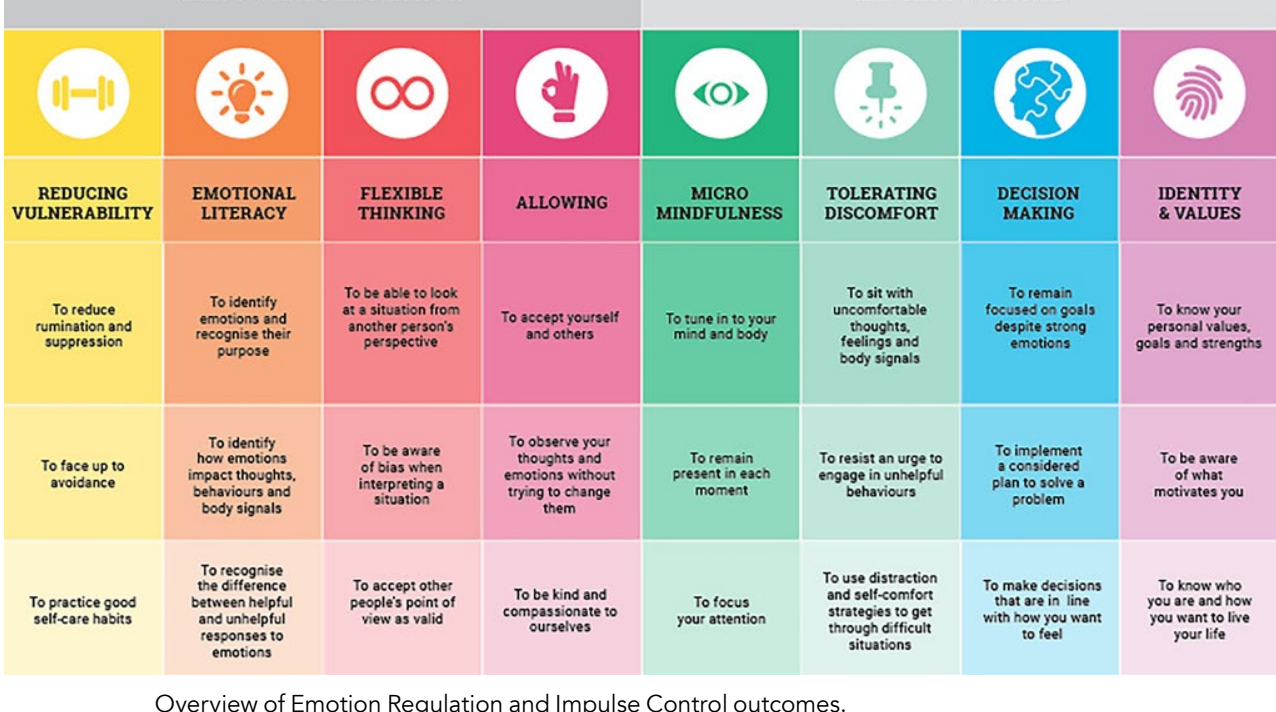

ERIC was designed to promote healthy

social and emotional development outcomes for vulnerable young people.

emotional clarity, everyday mindfulness, problem solving, values-based decision

making, and goal-directed behaviour.

OPTIMISING HEALTH OUTCOMES The eight domains the ERIC programm is organised into each pertain to

important processes underpinning

healthy Emotion Regulation and Impulse Control. Firstly, Reducing Vulnerability helps young people to reduce rumination and emotional suppression and to face up to avoidance by teaching strategies that interupt ruminative thinking cycles, reaching out to others, eating, sleping and exercising well and being kind to the self and others. The second module focuses on Emotional Literacy, and how to identify emotions and understand the way these impact thoughts, behaviours, and the body, as well as recognise their purpose. Emotional literacy also includes being able to recognise the difference between helpful and unhelpful responses to emotions. Thirdly, the development of Flexible Thinking is taught by helping young people learn to look at a situation from another person's perspective, be aware of bias when interpreting situation and to learn to accept other people's perspectives as valld. The fourth modula Allowing, incorporates acceptance of the
self and others, including observing and people who would not ordinaly undertook a number of research trials. The results of these trials have been published in numerous scientif papers eporting on the effectiveness of ERIC in ddressing the mental health needs of complex health needs. One study, delivered as an adjunct to residential substance use treatment found a $60 \%$ reduction of emotion dysregulation and reductions of $50-60 \%$ in depression and anxiety post-intervention. In another study with participants accessing youth mental health support senvices, research indicated clear associations between emotion regulation responses and symptoms of psychopathology when adaptive strategies were absent. Another twelve-week ERIC intervention programme for young people, alongside usual care, showed an improvement in derion dystegulation and symptoms of depression, anxiety and stress.

Unlike the large majority of clinical trials in young people which are egularly criticised due to their lack of studies have been undertaken in partnership with clinical services and therefore have included samples of youn people that are highly vulnerable and arguably more representative of actua senvice users.

\section{HOPE FOR A BETTER TOMORROW} Vulnerable young people may experience ongoing individual and psychosocial vulnerability throughout their life span, if not correctly supported. Drs Hall and Sloan say that ERRC was designed to promote healthy social and emotiona young people. The ability to effectively regulate emotions, manage uras and make good decisions in spite emotional distress are essential in helping young people to navigate the immense social and emotional demands of this period. The researchers explain that can help young people to navigate the different domains of their life, which include the physical, social, emotional, academic, moral, cultural, spiritual, sexual, and intellectual sphere. Learning how to manage and regulate emotions and behavioural impulses empowers young people to create a sense of emotional safety and protection for themselves through challenging situations.

\section{Behind the Research Ororke \\ W: www.deakin.edu.au/about-deakin/people/kate-hall

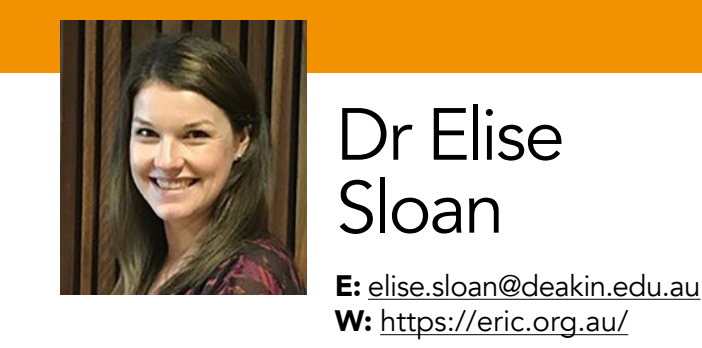

Research Objectives

Dr Hall and Dr Elise Sloan collaborated on a six-year body of research that underpinned the development of the Emotion

Regulation Impulse Control (ERIC) intervention, aiming to improve the social and emotional outcomes of vulnerable young people.

\section{Detail} populations, all of the ERIC reesearch a healthy development in these areas

School of Psychology, Faculty of Health,

221 Burwood Highway, Burwood,

VIC 3125
Australia

Bio

Dr Kate Hall is a Clinical Psychologist and Senior Lecturer at Deakin University, Melbourne, Australia. Her research focuses on the role of emotion dysregulation as a transdiagnostic treatment target for vulnerable young substance use iss developer of the Emotion Regulation She conducts translational research in partnership with services to improve practices aimed at improving social young people.

Dr Elise Sloan is an early-career researcher and Clinical Psychologis Dr Sloan has a track record in clinical research, with an interest in dysregulation plays in vulnerable young the dissemination of evidence informed

people who present with complex

pealth and substance use needs.

Funding

Helen Macpherson Smith Trust; Queensland Department of Justice and Attorney Gen

\section{Collaborators}

Petra K. Staiger, George Youssef, Angela Simpson, Natasha Perry, Richard Moulding, Amanda L. Baker, Alison Dunlop

\section{References} - Hall, K., Youssef, G., Simpson, A., Sloan, E., Graeme, L., Perry,
N., Moulding, R., Baker, A. L., Beck A. \& K., Staiger, P. K. (2021). An Emotion Regulation and Impulse Control (ERIC) Intervention for Vulnerable Young People: A Multi-Sectoral Pilot Study.
Frontiers in Psychology, 12. Available at: https://doi.org/10.3389/
fpsyg.2021.554100 - Hall, K., \& Simpson, A. (2018). ERIC manual version II: helping young people regulate their emotions and control impulsive behaviours. Deakin Univers

- Sloan, E., Hall, K., Moulding, R., Bryce, S., Mildred, H., \& Staiger, P. K. (2017). Emotion regulation as a transdiagnostic treatment construct across anxiety, depression, substance, eating and Psychology Review, 57, 141-163. Available at: https./dai. org/10.1016/i.cpr.2017.09.002 Stoan, E., Hall, K., Youssel, G.J., Moulding, R., Mildred, H. \& Staiger, P.K. (2019). Profiles of Emotion Regulation in Young People Therapy and Research. Available at https///doiorg/10.1007/s 10608019-10003-4 within a real-world setting, wh and Sloan and heach tials, Drs Hal
S.,
H., \& Staiger, P. .'. (2018). An emotion regulation treatment for young people with complex substance use and mental health issues A case series analysis. Cognitive and Behavioural Practice, 25, 427-

\section{Personal Response}

\section{Would ERIC have applicability for all} Wdolescents as part of a Life Skills curriculum in

II ERIC has captured the interest of parents, II ERIC has captured the interest of parents, community-wide dissemination as part of a 'whole of community' approach in Melbourne, Australia. This three-year programme of work has meant family and youth practitioners have undergone ERIC throughout the community. Teachers at high school and primary schools, practitioners primary health services, youth drop-in centres and to a healthy community that cares for and supports

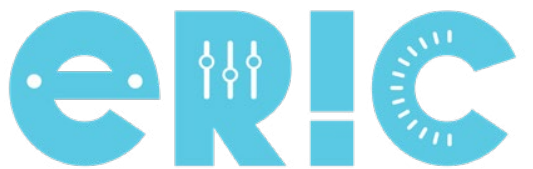
outreach services for families across an inner city catchment are being trained in ERS to contribute 